\title{
Large time behavior for a porous medium equation in a nonhomogeneous medium with critical density
}

\author{
Razvan Gabriel Iagar ${ }^{* \dagger}$ \\ Ariel Sánchez,
}

\begin{abstract}
We study the large time behavior of solutions to the porous medium equation in nonhomogeneous media with critical singular density

$$
|x|^{-2} \partial_{t} u=\Delta u^{m}, \quad \text { in } \mathbb{R}^{N} \times(0, \infty),
$$

where $m>1$ and $N \geq 3$. The asymptotic behavior proves to have some interesting and striking properties. We show that there are different asymptotic profiles for the solutions, depending on whether the continuous initial data $u_{0}$ vanishes at $x=0$ or not. Moreover, when $u_{0}(0)=0$, we show the convergence towards a profile presenting a discontinuity in form of a shockwave, coming from an unexpected asymptotic simplification to a conservation law, while when $u_{0}(0)>0$, the limit profile remains continuous. These phenomena illustrate the strong effect of the singularity at $x=0$. We improve the time scale of the convergence in sets avoiding the singularity. On the way, we also study the large-time behavior for a porous medium equation with convection which is interesting for itself.
\end{abstract}

AMS Subject Classification 2010: 35B33, 35B40, 35K10, 35K67, 35Q79.

Keywords and phrases: porous medium equation, non-homogeneous media, singular density, asymptotic behavior, radially symmetric solutions, nonlinear diffusion.

\section{Introduction}

The goal of this paper is to study the asymptotic behavior of solutions to the following porous medium equation in nonhomogeneous media with critical singular density:

$$
|x|^{-2} \partial_{t} u(x, t)=\Delta u^{m}(x, t), \quad(x, t) \in \mathbb{R}^{N} \times(0, \infty),
$$

\footnotetext{
*Dept. de Análisis Matemático, Universitat de Valencia, Dr. Moliner 50, 46100, Burjassot (Valencia), Spain, e-mail: razvan.iagar@uv.es

${ }^{\dagger}$ Institute of Mathematics of the Romanian Academy, P.O. Box 1-764, RO-014700, Bucharest, Romania.

${ }^{\ddagger}$ Departamento de Matemática Aplicada, Universidad Rey Juan Carlos, Móstoles, 28933, Madrid, Spain, e-mail: ariel.sanchez@urjc.es
} 
with $m>1$ and $N \geq 3$. An important feature of this equation is the influence of the density that is at the same time singular at $x=0$ and degenerate at infinity, giving rise to very interesting and unexpected results.

Equations of type (1.1) with general densities, more precisely

$$
\varrho(x) \partial_{t} u(x, t)=\Delta u^{m}(x, t), \quad(x, t) \in \mathbb{R}^{N} \times(0, \infty),
$$

where $\varrho$ is a density function with suitable behavior, have been proposed by Kamin and Rosenau in a series of papers [12, 13, 14 to model thermal propagation by radiation in non-homogeneous plasma. Afterwards, a huge development of the mathematical theory associated to Eq. (1.2) begun, usually under conditions such as

$$
\varrho(x) \sim|x|^{-\gamma}, \quad \text { as }|x| \rightarrow \infty,
$$

for some $\gamma>0$, as for example in the following papers [3, 4, 20, 24, 21, 22, 11] where its qualitative properties and asymptotic behavior are studied. In particular, along these references, the basic existence and regularity properties are proved under suitable conditions for the initial data, and a detailed study for the asymptotic behavior for $\gamma \neq 2$ has been done. Thus, it has been noticed that for $\gamma \in(0,2)$, the solutions have similar qualitative properties to the ones of the standard porous medium equation

$$
u_{t}=\Delta u^{m}
$$

see [20, 22], while for $\gamma>2$ they are quite different [11]. Thus, the value $\gamma=2$ is critical. A first step in the study of Eq. (1.2) with $\varrho(x) \sim|x|^{-2}$ at infinity, but $\varrho$ regular at $x=0$, has been done in the recent paper [18, having as starting point some conjectures and comments in [11].

On the other hand, concerning the asymptotic behavior of solutions to Eq. (1.2), it is shown that the profiles are special solutions of Eq. (1.1), giving thus rise to the natural problem of the study of the pure power density case $\varrho(x)=|x|^{-\gamma}$. A special feature of Eq. (1.1), besides its general interest for classifying asymptotic profiles for the general case (1.2), is the fact that a strong singularity appears at $x=0$. As we will see, the presence of this singular coefficient (in contrast with the above mentioned papers where $\varrho(x)$ is supposed regular at $x=0$ ), introduces various unexpected mathematical phenomena, as the appearance of two regimes of convergence, different profiles for different initial data only near $x=0$, and backward evolution of the profiles.

Recently, in a previous work [10], the authors proved some of these interesting and striking features for the easier case of the linear equation

$$
|x|^{-2} \partial_{t} u(x, t)=\Delta u, \quad(x, t) \in \mathbb{R}^{N} \times(0, \infty),
$$

where all the profiles are explicit and one can use the theory of the heat equation. Moreover, a formal study of the radially symmetric solutions to Eq. (1.1) for general $\gamma$ has been performed in [9], including some mappings that will be useful in the sequel for the case $\gamma=2$.

Before stating and explaining our main results, we want to mention that we only consider dimensions $N \geq 3$, letting apart the cases $N=1$ and $N=2$ for a further work, due to some differences with respect to the general functional theory. 
Main results. In the present paper, we deal with the Cauchy problem associated to Eq. (1.1) with initial condition

$$
u_{0}(x):=u(x, 0) \in L_{2}^{1}\left(\mathbb{R}^{N}\right), \quad u_{0} \geq 0,
$$

where $N \geq 3$ and, as usual,

$$
L_{2}^{1}\left(\mathbb{R}^{N}\right):=\left\{h: \mathbb{R}^{N} \mapsto \mathbb{R}, h \text { measurable, } \int_{\mathbb{R}^{N}}|x|^{-2} h(x) d x<\infty\right\} .
$$

In Section 2 we make a review of the notion of strong solution to (1.1) and the wellposedness results we need. In particular, for any initial condition $u_{0}$ as above, there exists a strong solution $u$ having some additional qualitative properties, see Theorem 2.1 .

As it will become clear from the subsequent analysis, there is a branching point for the large-time behavior of general solutions to (1.1). Similar to the linear case $m=1$ studied in our previous paper [10], a big difference is related to whether $u_{0}(0)=0$ or $u_{0}(0) \neq 0$. But let us state rigorously our results.

1. Initial data $u_{0}$ such that $u_{0}(0)=0$. Let us introduce the following weighted norm:

$$
\|h\|_{p, N}=\int_{\mathbb{R}^{N}}|x|^{-N}|h(x)|^{p} d x
$$

We state first a simple convergence result in the previous integral norm, which is interesting by itself but can be also seen as a preliminary.

Theorem 1.1. Let $u$ be a radially symmetric solution to Eq. (1.1) with initial condition $u_{0}$ satisfying (1.5) and furthermore

$$
u_{0}(0)=0, \quad M_{u_{0}}:=\int_{\mathbb{R}^{N}}|x|^{-N} u_{0}(x) d x<\infty .
$$

Then, for any $1 \leq p<\infty$, we have

$$
\lim _{t \rightarrow \infty} t^{(p-1) / m p}\|u(t)-F(t)\|_{p, N}=0,
$$

where

$$
F(x, t)= \begin{cases}0, & \text { for }|x|<e^{-k t^{1 / m}}, \\ t^{-1 / m}\left[-\frac{1}{m(N-2)} \log |x| t^{-1 / m}\right]_{+}^{1 /(m-1)}, & \text { for }|x| \geq e^{-k t^{1 / m}},\end{cases}
$$

and $k=k\left(M_{u_{0}}\right)$ has a precise value depending on the mass $M_{u_{0}}$ above.

We notice that the previous result fails to hold in the $L^{\infty}$ norm, since the limit is discontinuous (is a shockwave). In order to improve this result and give a more precise description, we use the convergence in the sense of graphs for multivalued functions. This is a situation arising in cases of convergence towards discontinuous solutions of conservation laws, as explained for example in [5]. Before stating the result, we introduce the necessary elements, adapted to our case, in the following: 
Definition 1.2. Let $f, g: D \subseteq \mathbb{R} \mapsto 2^{\mathbb{R}}$ be two multivalued functions. We define the distance between their graphs in the natural way:

$$
d_{g}(f(x), g(x))=\inf \{|y-z|: y \in f(x), z \in g(x)\}, \quad \text { for any } x \in D .
$$

Let $\left\{f_{k}\right\}: D \subseteq \mathbb{R} \mapsto 2^{\mathbb{R}}$ be a sequence of multivalued functions and $F: D \subseteq \mathbb{R} \mapsto 2^{\mathbb{R}}$. We say that $\left\{f_{k}\right\}$ converges to $F$ in the sense of graphs if for any $\varepsilon>0$, there exists $k_{\varepsilon}$ sufficiently large such that

$$
d_{g}\left(f_{k}(x), F(x)\right) \leq \varepsilon, \quad \text { for any } k \geq k_{\varepsilon}, x \in D .
$$

We notice that this notion of convergence generalizes the standard uniform convergence, to which it reduces if all the functions involved are univalued. In the case of a function $F$ having a jump discontinuity at some point $x_{0} \in D$, assuming that

$$
l_{-}=\lim _{x \rightarrow x_{0}^{-}} F(x)<\lim _{x \rightarrow x_{0}^{+}} F(x)=l_{+},
$$

we will think at it as the multivalued map with $F\left(x_{0}\right)=\left[l_{-}, l_{+}\right]$(and similarly if $l_{-}>l_{+}$) and $F(x)$ as usual at $x \neq x_{0}$.

In order to apply it in our case, as we only deal with radially symmetric solutions, we introduce the new variables and functions

$$
\bar{u}(y, t)=t^{1 / m} u(|x|, t), \quad \bar{F}(y, t)=t^{1 / m} F(|x|, t), \quad y=-\log |x| t^{-1 / m} .
$$

Notice that in the new variables, the profile $\bar{F}(y, t)=[y / m(N-2)]^{1 /(m-1)}$ on its support, thus it is stationary, and presents a unique jump discontinuity at $y=k$. With the considerations above, we have the following result

Theorem 1.3. Let $u_{0}, u, F$ as in Theorem 1.1. Then, in the notation of (1.9), we have

$$
\bar{u}(y, t) \longrightarrow \bar{F}(y), \quad \text { as } t \rightarrow \infty,
$$

the convergence being in the sense of graphs.

Observe that this convergence implies uniform convergence in the form

$$
\lim _{t \rightarrow \infty} t^{1 / m}\left|u\left(e^{t^{1 / m} \log |x|}, t\right)-F\left(e^{t^{1 / m} \log |x|}, t\right)\right|=0
$$

away from the shock line of $\bar{F}$, and a control of the maxima of the family $\bar{u}(t)$ at the shock line $y=k$. We discover thus a striking phenomenon: convergence to a discontinuous profile presenting a shock line, which usually comes from a conservation law. But in Eq. (1.1), it is not obvious which internal process of it may give rise to such discontinuous profile.

We represent in Figure 1 both the asymptotic profile $F$ and the evolution of a solution, showing the formation of the shockwave. The numerical experiment has been done for $u_{0}(x)=\max \{(x-0.5)(1.5-x), 0\}$.

We remark that in the second figure, some tail appears in the evolution. The explanation for it is the following: in the global time-scale, this tail for $|x|>1$ is negligible in the largetime limit (it will tend uniformly to 0). But refining the scale, one can see some different localized behavior close to infinity, as shown in Theorem 1.9 below.

For general (not necessarily radially symmetric) solutions, we can get the optimal time decay rate applying the comparison principle. 

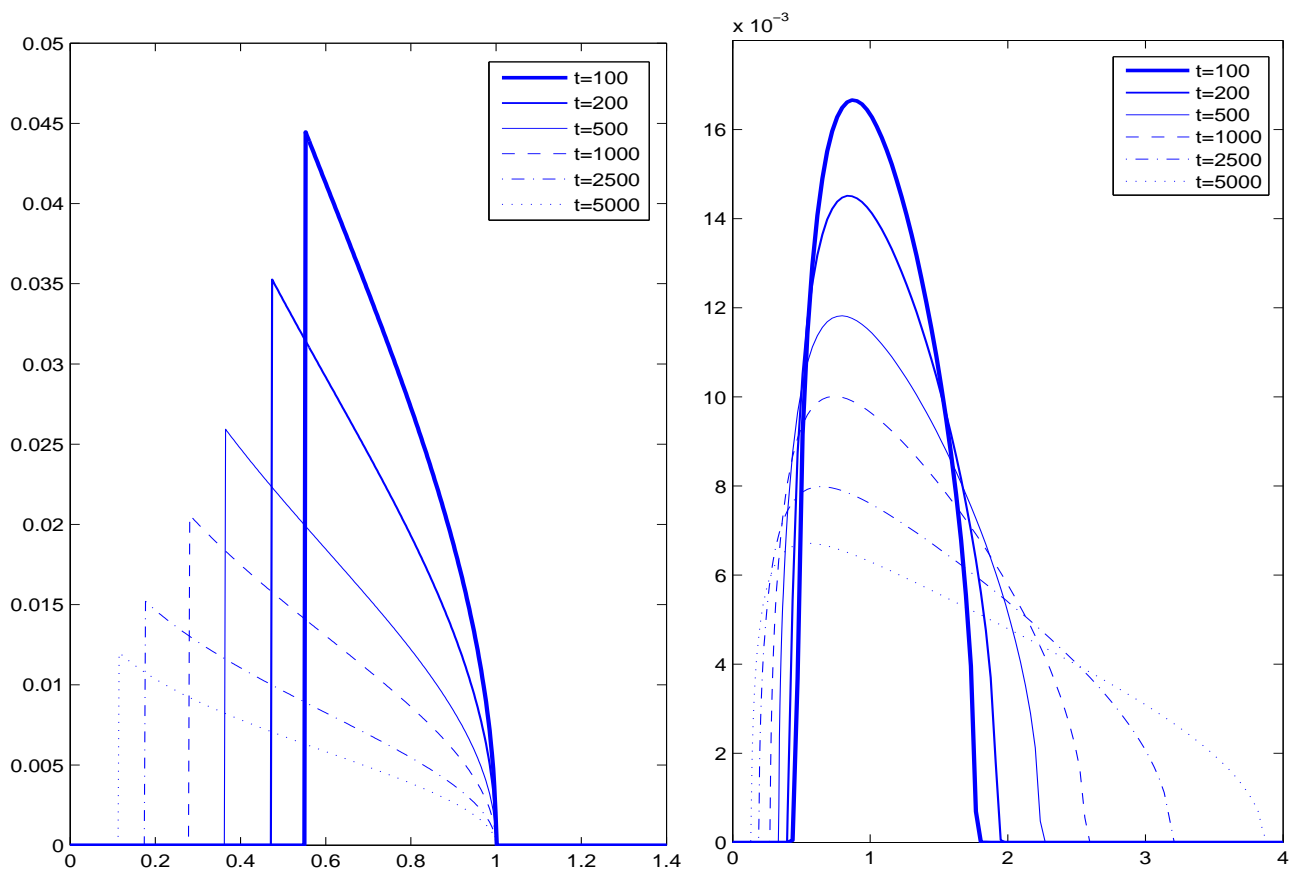

Figure 1: Profile $F$ (left) and evolution of a general solution (right) for $m=3$ in dimension $N=3$.

Corollary 1.4. Let $u$ be a general solution with initial condition $u_{0} \in L^{\infty}\left(\mathbb{R}^{N}\right)$ and as in Theorem [1.1, such that there exists $r>0$ with

$$
\min _{|x|=r} u_{0}(x)>0 .
$$

Then, the optimal time decay rate of $\|u(t)\|_{\infty}$ is $t^{-1 / m}$.

2. Initial data $u_{0}$ such that $u_{0}(0)=K>0$. In this case, we can give a general result, showing that the value $K$ at the origin is preserved along the evolution, and that we have a uniform convergence in the standard sense towards a continuous profile. We state first the result for radially symmetric functions, as follows

Theorem 1.5. Let $u$ be a radially symmetric solution to Eq. (1.1) with initial condition $u_{0}$ satisfying (1.5), $u_{0}(0)=K>0$ and furthermore

$$
u_{0} \text { continuous at } \mathrm{x}=0, \quad 0 \leq u_{0}(x) \leq K, \text { for any } x \in \mathbb{R}^{N}, \quad \lim _{|x| \rightarrow \infty} u_{0}(x)=0,
$$

Then, we have

$$
\lim _{t \rightarrow \infty}\left|u(x, t)-E_{K}(x, t)\right|=0
$$


uniformly in $\mathbb{R}^{N}$, where

$$
E_{K}(x, t)= \begin{cases}K, & \text { if } 0 \leq|x| \leq e^{-m K^{m-1}(N-2) t}, \\ {\left[-\frac{1}{m(N-2)} \frac{\log |x|}{t}\right]^{1 /(m-1)},} & \text { if } e^{-m K^{m-1}(N-2) t}<|x|<1, \\ 0, & \text { if }|x| \geq 1\end{cases}
$$

In particular, we also have that $u(0, t)=K$, for any $t>0$.

The limit profiles obtained in Theorems 1.1 and 1.5 are interesting and difficult to guess at first sight. Indeed, they are apparently not related to the equation (1.1) itself, but they are obtained via an asymptotic simplification process applied to some equation of porous medium type with convection, in which (1.1) can be mapped via a transformation indicated in Section 3 ,

In the general case of non-radially symmetric solutions, we need to impose an estimate of the decay at infinity. More precisely, we have:

Theorem 1.6. Let $u$ be a general solution to Eq. (1.1), with initial condition $u_{0}$ satisfying (1.5), $u_{0}(0)=K>0$, (1.12) and furthermore, that there exist $\delta>0$ small and $R>0$ large such that

$$
u_{0}(x) \leq|x|^{2-N-\delta}, \quad \text { for }|\mathrm{x}|>\mathrm{R} .
$$

Then, (1.13) holds true uniformly in $\mathbb{R}^{N}$, with the same profile $E_{K}$ as in Theorem 1.5 .

Remark 1.7. Condition (1.15) in Theorem 1.6 can be made slightly more general in the following form: there exists a function $\Psi: \mathbb{R} \rightarrow[0, \infty)$ and some $R>0$ such that

$$
u_{0}(x) \leq \Psi(|x|), \quad \text { for any } x \in \mathbb{R}^{N},|x|>R, \quad \text { and } \int_{R}^{\infty} r^{N-3} \Psi(r) d r<\infty .
$$

We represent in Figure 2 both the profile $E_{K}$ and the evolution of a general solution, showing how its form approaches the expected one. There is again a problem with the tails for $|x|>1$, as the outer time-scale is different from the global one. This will be explained below in Theorem 1.9.

Let us notice some curious facts resulting from our analysis.

Remark 1.8 (Lack of continuity as $m \rightarrow 1$ ). We have discovered a striking phenomenon: there is a lack of "continuity" as $m \rightarrow 1$ in our equation. Indeed, when considering the standard porous medium equation (1.3) and the heat equation, one notices that there appears some kind of "continuity" as $m \rightarrow 1$, at some formal level; just as an example, the optimal

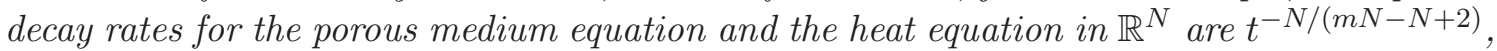
respectively $t^{-N / 2}$, and they are obviously continuous as $m \rightarrow 1$; the same happens about the similarity exponents of the fundamental solutions of both equations.

Recalling the analysis done for the case $m=1$, that is

$$
|x|^{-2} u_{t}=\Delta u,
$$

in our previous paper [10], we notice that the above considerations are not true for Eq. (1.1) with $m \geq 1$ : the optimal decay rates (when dealing with initial data $u_{0}(0)=0$ ) are $t^{-1 / m}$ for $m>1$, respectively $t^{-1 / 2}$ for $m=1$ (see [10]). 

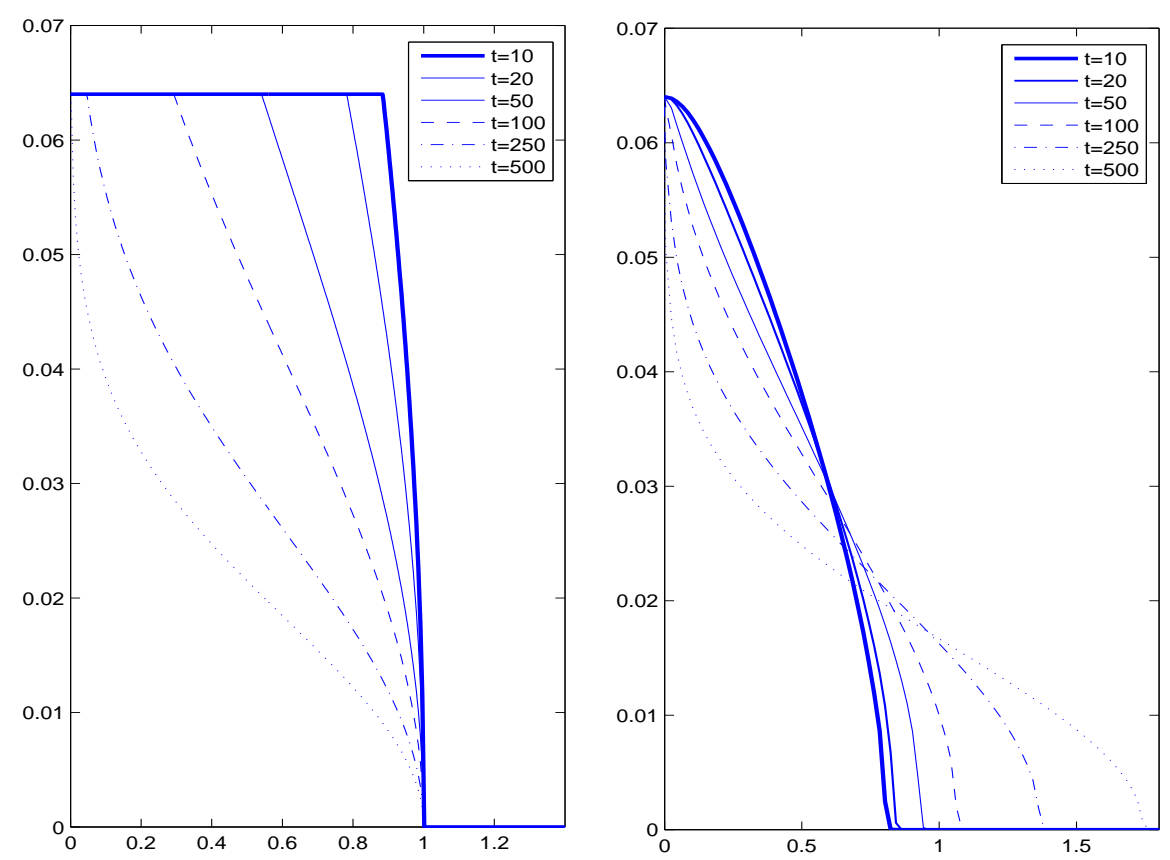

Figure 2: Profile $E_{K}$ and evolution of a general solution for $m=3$ in dimension $N=3$.

This is very surprizing, and the explanation is the following: Eq. (1.1) can be mapped, for all $m \geq 1$, by the transformation (3.1), into the general convection-diffusion equation

$$
w_{\tau}=\left(w^{m}\right)_{s s}-\left(w^{m}\right)_{s}, \quad \text { in } \mathbb{R} \times(0, \infty) .
$$

But the properties of it for $m>1$ depart strongly from the ones of the linear case $m=1$, as shown in [5, 17]. This explains our apparently strange "lack of continuity" in the exponents.

3. Finer asymptotics in the "outer" region. We can improve the previous convergence results in regions that are far away from the origin by refining the time-scale (making a "zoom" on that region), as also the numerical experiments above had shown by the appearance of thin tails for $|x|>1$ at any $t>0$. We recall that there exists a family of explicit solutions to Eq. (1.1):

$$
B_{D}(x, t)=t^{-1 /(m-1)}\left[D-\frac{1}{m(N-2)} \log \left(|x| t^{-1 /(m-1)(N-2)}\right)\right]_{+}^{1 /(m-1)} \quad, \quad \text { for any } D>0
$$

which does not enter into the framework of our previous study, since it is singular at $x=0$. But it gives a better information with respect to large time behavior and decay rates in regions that are far from the origin; more precisely, we have 
Theorem 1.9. Let $u$ be a solution to Eq. (1.1) with initial datum $u_{0}$ satisfying (1.5). Then, for any $\delta>0$, we have

$$
\lim _{t \rightarrow \infty} t^{1 /(m-1)}\left|u(t)-B_{D}(t)\right|=0, \quad \text { uniformly in the set }\left\{|x| \geq \delta t^{1 /(m-1)(N-2)}\right\},
$$

where $D$ is the (unique) constant in (1.16) such that $\left\|B_{D}\right\|_{L_{2}^{1}}=\left\|u_{0}\right\|_{L_{2}^{1}}$.

The proof is a straightforward adaptation of the one of [18, Theorem 1.1], as all the technical steps there are done in compact sets of $\mathbb{R}^{N} \backslash\{0\}$ where the singularity at $x=0$ does not have any effects. We omit the details.

Notice that Theorem [1.9 gives an optimal information in outer sets of the type $\{|x| \geq$ $\left.C t^{1 /(m-1)(N-2)}\right\}$, where our analysis was only giving a non-optimal decay rate due to the domination (in the global profiles) of the inner regions close to the origin. A similar situation was described in [11, Section 7].

Organization of the paper. In Section 2 we give the notion of a solution to (1.1) and we recall some well-posedness results proved in [18. Section 3 deals with a transformation mapping the radially symmetric solutions to (1.1) to solutions to a porous medium equation with convection that is also interesting in itself. Based on this transformation, we prove Theorems 1.1 and 1.3 in Section 4 using previous knowledge on the transformed equation. The proof of 1.5 is done in Section 5 and relies on the four-step technique, which is a general method in proving large time behavior for nonlinear diffusion equations, see [25, 19, 18] for other examples of use; the asymptotic simplification will arise then in a natural way. Then, passing to general solutions and proving Theorem 1.6 and Corollary 1.4 is an application of the comparison principle, see Section 6. We finally include a Section 7 on further extensions to other equations or densities of interest and some open problems.

\section{Well-posedness. Functional preliminaries}

In this section we make precise our notions of solution that we use along the present paper. We deal with the Cauchy problem

$$
\begin{cases}|x|^{-2} u_{t}=\Delta u^{m}, & \text { in } \mathbb{R}^{N} \times(0, \infty), \\ u(x, 0)=u_{0}(x), & x \in \mathbb{R}^{N}\end{cases}
$$

with $u_{0}$ satisfying (1.5). The theory of existence and uniqueness for this Cauchy problem has been recently studied in [18, from where we extract some statements for the sake of completeness. Let

$$
Q^{*}:=\mathbb{R}^{N} \times(0, \infty) \backslash\{(0, t): t>0\} .
$$

We do not insist on the notion of weak solution to (1.1), which is a straightforward adaptation of [18, Definition 2.1] to our special density $\varrho(x)=|x|^{-2}$. We next consider the class $\mathcal{F}$ of functions $u \in C\left([0, \infty) ; L_{2}^{1}\right) \cap C\left(Q^{*}\right)$ satisfying the following limitations on their behavior near $x=0$ and close to infinity: for every $\tau>0$, there exists a constant $C(\tau)$ such that

$$
u(x, t) \leq C(\tau)|\log x|^{1 /(m-1)}, \quad \text { for any } t>\tau, 0<|x|<\varepsilon<1
$$

for some $\varepsilon>0$ sufficiently small, and

$$
u(x, t) \leq C(\tau), \quad \text { for any } t>\tau,|x|>1 .
$$


With these conditions, we have the following

Theorem 2.1. Let $u_{0}$ as in (1.5). Then the Cauchy problem (2.1) admits a unique (weak) solution $u \in \mathcal{F}$ such that

$$
|x|^{-2} u_{t}, \Delta u^{m} \in L_{\mathrm{loc}}^{1}\left(Q_{*}\right), \quad|x|^{-2} u_{t}=\Delta u^{m} \text { a.e. in } Q_{*} .
$$

This theorem is proved in [18, Theorem 3.1]. Following previous literature, a solution $u \in \mathcal{F}$ satisfying (2.2) will be called a strong solution in the sequel. Moreover, if $u_{0}$ is continuous, then the solution $u$ is continuous including at the origin.

We end these preliminaries with the following contraction principle, also proved in [18], that implies both uniqueness and comparison:

Proposition 2.2 ( $L_{2}^{1}$-Contraction principle). Let $u_{1}, u_{2}$ be two strong solutions of Eq. (1.1). For $0<t_{1}<t_{2}$ we have

$$
\int_{\mathbb{R}^{N}}|x|^{-2}\left[u_{1}\left(x, t_{2}\right)-u_{2}\left(x, t_{2}\right)\right]_{+} d x \leq \int_{\mathbb{R}^{N}}|x|^{-2}\left[u_{1}\left(x, t_{1}\right)-u_{2}\left(x, t_{1}\right)\right]_{+} d x,
$$

where $[g]_{+}$represents the positive part of a generic function $g$.

\section{Radially symmetric solutions. The transformation}

We now restrict ourselves to radially symmetric solutions $u(x, t)=u(r, t), r=|x|$, to (1.1). We introduce the following change of variables:

$$
u(r, t)=w(s, \tau), \quad r=e^{\theta s}, t=\theta^{2} \tau,
$$

for some $\theta$ to be chosen later. Denoting by subindex the derivative with respect to the corresponding variable, we notice that

$$
\left(u^{m}\right)_{r}(r, t)=\frac{1}{\theta r}\left(w^{m}\right)_{s}(s, \tau), \quad\left(u^{m}\right)_{r r}(r, t)=\frac{1}{(\theta r)^{2}}\left(w^{m}\right)_{s s}(s, \tau)-\frac{1}{\theta r^{2}}\left(w^{m}\right)_{s}(s, \tau) .
$$

Replacing these formulas into the radially symmetric form of Eq. (1.1), that is

$$
r^{-2} \partial_{t} u(r, t)=\left(u^{m}\right)_{r r}(r, t)+\frac{N-1}{r}\left(u^{m}\right)_{r}(r, t),
$$

we arrive to the following equation satisfied by $w$ :

$$
\partial_{\tau} w(s, \tau)=\left(w^{m}\right)_{s s}(s, \tau)+(N-2) \theta\left(w^{m}\right)_{s}(s, \tau) .
$$

As we are in dimension $N \geq 3$, we arrive to a porous medium equation with convection in one space dimension. We can simply choose then $\theta=-1 /(N-2)<0$ to get

$$
\partial_{\tau} w(s, \tau)=\left(w^{m}\right)_{s s}(s, \tau)-\left(w^{m}\right)_{s}(s, \tau) .
$$

The equation (3.3) is very interesting by itself and it has been obtained from the modelling of the transport of a solute through a porous medium, the physical models appearing in [7] and references therein. Starting from this point, the mathematical theory for (3.3) 
(and also for the one with a plus sign in the right hand side, which is equivalent to (3.3) changing $s$ into $-s$ ) developed rapidly in the framework of the so-called mild solutions, generated via semigroup theory, as for example in [1, 2, 6] and references therein. In particular, well-posedness for the Cauchy problem is proved when $w_{0} \in L^{1}(\mathbb{R})$. The large-time behavior of solutions to (3.3) has been studied by Laurençot and Simondon in [16, 17], where again they ask for the initial data $w_{0}$ to be integrable.

Remark. The same transformation applies in dimension $N=2$ leading to the standard porous medium equation (1.3). We do not deal with this case here, as explained in the Introduction.

\section{Radially symmetric solutions. Asymptotic behavior when $u_{0}(0)=0$}

In this section we prove Theorems 1.1 and 1.3, We will work with the convection-diffusion equation (3.3) obtained via the transformation (3.1). A specific fact in this type of equations is the competition between the two processes (nonlinear diffusion and convection), leading to different ranges where the large time behavior is very different. In our case, it is shown in [17] that we are in the range where the convection process is dominant.

Before beginning the rigorous proof, we have to "guess" the correct profile. This shows how the asymptotic simplification comes into play, once we make the ansatz:

$$
w(s, \tau)=\tau^{-1 / m} v(y, \tau), \quad y=s \tau^{-1 / m} .
$$

Notice that the ansatz is coherent with our case, of initial condition vanishing at the origin (that is, after the transformation (3.1), that $\lim _{s \rightarrow \infty} w(s, 0)=0$ ), as we expect to have a time decay. Thus, we calculate

$$
w_{\tau}(s, \tau)=\tau^{-1 / m} v_{\tau}(y, \tau)-\frac{1}{m} \tau^{-1-1 / m}\left(v(y, \tau)+y v_{y}(y, \tau)\right)
$$

and

$$
\left(w^{m}\right)_{s}(s, \tau)=\tau^{-1-1 / m}\left(v^{m}\right)_{y}(y, \tau), \quad\left(w^{m}\right)_{s s}(s, \tau)=\tau^{-1-2 / m}\left(v^{m}\right)_{y y}(y, \tau),
$$

hence, letting also $\bar{\tau}=\log (1+\tau)$, we deduce that $v=v(y, \bar{\tau})$ solves

$$
v_{\bar{\tau}}=\frac{1}{m}\left(v+y v_{y}\right)-\left(v^{m}\right)_{y}+e^{-\bar{\tau} / m}\left(v^{m}\right)_{y y} .
$$

Letting formally $\bar{\tau} \rightarrow \infty$ in (4.2) and assuming that the time decay in (4.1) is the correct one, we expect to converge to a stationary solution $v^{*}=v^{*}(y)$ solving

$$
\frac{1}{m}\left(y v_{y}^{*}+v^{*}\right)-\left[\left(v^{*}\right)^{m}\right]_{y}=0 .
$$

By integration and taking into account that $\lim _{y \rightarrow \infty} v^{*}(y)=0$, we find $v^{*}(y)=(y / m)_{+}^{1 /(m-1)}$, hence, coming back to the original variables, we expect that the limit profile of $w$ as $\tau \rightarrow \infty$ will be

$$
W(s, \tau)= \begin{cases}\tau^{-1 / m}\left(\frac{1}{m} s \tau^{-1 / m}\right)^{1 /(m-1)}, & \text { for } s \in\left[0, k \tau^{1 / m}\right), \\ 0, & \text { otherwise }\end{cases}
$$


which coincides with the one in [17, Theorem 1.4] and is a special solution to the following first order equation obtained via asymptotic simplification

$$
W_{\tau}+\left(W^{m}\right)_{s}=0 .
$$

The constant $k$ for the branching point above is unique and is chosen for such profile to have initial mass $M_{u_{0}}$. Finally, in the initial equation, the limit profile writes

$$
F(x, t)= \begin{cases}0, & \text { for }|x|<e^{-k t^{1 / m}} \\ t^{-1 / m}\left[-\frac{1}{m(N-2)} \log |x| t^{-1 / m}\right]_{+}^{1 /(m-1)}, & \text { for }|x| \geq e^{-k t^{1 / m}}\end{cases}
$$

as expected in Theorem [1.1.

All the previous calculations were totally formal; they show how the limit profile appears in a logical manner in our work. We are now in position to provide the rigorous proofs.

Proof of Theorem 1.1. This is simple in view of the results in [17]. Let $u_{0}$ be an initial condition as in the statement of Theorem 1.1, $u$ the (radially symmetric) solution to Eq. (1.1) with initial condition $u_{0}$ and $w$ be the solution to Eq. (3.3) obtained from $u$ via the tranformation (3.1). Then, condition (1.6) implies that $\lim _{s \rightarrow \infty} w_{0}(s)=0$ and that

$$
\int_{-\infty}^{\infty} w_{0}(s) d s=(N-2) \int_{0}^{\infty} \frac{u_{0}(r)}{r}=\frac{(N-2) M_{u_{0}}}{\omega_{1}}<\infty
$$

where $\omega_{1}$ is the area of the unit sphere in $\mathbb{R}^{N}$. It follows that $w_{0} \in L^{1}(\mathbb{R})$. We are in the same conditions as in [17, Theorem 1.4] (for the case $q=m$ in the notations used there), hence we deduce that, for any $p \in[1, \infty)$, we have

$$
\lim _{t \rightarrow \infty} t^{(p-1) / m p}\|w(t)-W(t)\|_{p}=0,
$$

which, undoing the change of variables, yields (1.7).

Proof of Theorem 1.3. In order to prove the convergence in the sense of graphs, we use the following result, which is an adaptation of [5, Lemma 2.2].

Lemma 4.1. Let $g \in L^{1}(\mathbb{R})$ be a nonnegative function such that

$$
\left(g^{m-1}\right)_{x} \leq 1, \quad \text { for any } x>0 .
$$

Consider the function $G$ defined as

$$
G(x)= \begin{cases}x^{1 /(m-1)}, & \text { for } 0 \leq x \leq T \\ 0, & \text { otherwise }\end{cases}
$$

where $T$ is chosen such that $\|G\|_{1}=\|g\|_{1}$. Assume that $\|g-G\|_{1}<\varepsilon$ for some $\varepsilon>0$. Then the distance between the graphs of $g$ and $G$ can be estimated by a positive power of $\varepsilon$. 
Notice that in [5, Lemma 2.2] there is a weaker integral condition, which is implied by our condition on the distance in $L^{1}$, and the lemma is more general, holding true for any $k>0$ instead of $m-1$.

We want to apply it for $g=w$ solution to Eq. (3.3) and $G=W$, the limit profile in (4.3). In order to do it, we need to prove that its conditions are fulfilled. The following result, which is interesting by itself, will imply (4.5).

Lemma 4.2. Let $w$ be a solution to (3.3) with initial condition $w_{0} \in L^{1}(\mathbb{R}) \cap L^{\infty}(\mathbb{R})$. Then

$$
\left(w^{m-1}\right)_{s} \leq \frac{1}{m \tau}
$$

Proof. We use a Bernstein technique. At a formal level, consider

$$
z(s, \tau):=\frac{m}{m-1} w(s, \tau)^{m-1} .
$$

Then by straightforward calculations (see [19]), the equation satisfied by $z$ is

$$
z_{\tau}=(m-1) z z_{s s}+z_{s}^{2}-(m-1) z z_{s} .
$$

Let then $p=z_{s}$. By differentiating in (4.7), we obtain the equation solved by $p$ :

$$
p_{\tau}=(m+1) p p_{s}+(m-1) z p_{s s}-(m-1) p^{2}-(m-1) z p_{s} .
$$

Let then $\bar{p}(\tau)=1 /(m-1) \tau$. We notice that $\bar{p}$ is a solution to (4.8) and $\bar{p}(s, 0)=+\infty>$ $p(s, 0)$ for any $s \in \mathbb{R}$. Thus, $\bar{p}$ is a supersolution to our problem, whence by standard comparison we get (4.6).

The above is a formal calculation, that holds true rigorously for solutions that are uniformly positive. Thus, for a rigorous proof, we have to consider solutions having $0<$ $\varepsilon \leq w_{0}(s)$, for which all previous calculations apply, then approximate as $\varepsilon \rightarrow 0$. We omit the details as this last technical step is quite standard (see [5, Lemma 1.1], [17, Lemma 2.10], in the latter a fully detailed proof of such approximation being given).

We are now in position to check the conditions in Lemma 4.1. Consider the new function and variable

$$
\bar{w}(y, \tau)=\tau^{1 / m} w\left(s \tau^{-1 / m}, \tau\right), \quad y=s \tau^{-1 / m}
$$

and notice that, for any $\tau>0$, we have

$$
\frac{\partial}{\partial y} \bar{w}^{m-1}(y, \tau)=\tau \frac{\partial}{\partial s} w^{m}\left(s \tau^{-1 / m}, \tau\right) \leq \frac{1}{m} .
$$

In the new variables $(y, \tau)$, we apply Lemma 4.1 for the following functions:

$$
g(y, \tau)=m^{1 /(m-1)} \bar{w}(y, \tau), \quad G(y, \tau)=m^{1 /(m-1)} \tau^{1 / m} W(y, \tau),
$$

where $W$ is the profile in (4.3). Notice that, in the new variables, $G$ has the required form, and the convergence in $L^{1}$ is insured by (4.4). The bound for $\left(g^{m-1}\right)_{y}$ follows from (4.9). Thus, an application of Lemma 4.1 gives that $\bar{w}$ converges to $W$ in the sense of graphs in the new variables. We end the proof by undoing the change of variables $(s, \tau) \mapsto(y, \tau)$ and transformation (3.1). 
Remark 4.3. Let us notice that, in the above proof, we show in particular that solutions $w$ to (3.3) satisfy

$$
\tau^{1 / m} w(y, \tau) \longrightarrow \tau^{1 / m} W(y, \tau), \quad y=s \tau^{-1 / m},
$$

with convergence in the sense of graphs. This is a slight improvement of [17, Theorem 1.4].

\section{Radially symmetric solutions. Asymptotic behavior when $u_{0}(0)=K>0$}

In this section we prove Theorem 1.5. Similar to the previous section, we begin with a formal calculation which will give us a guess of the profile. As we expect the value $K>0$ at the origin to maintain, no time decay is allowed in this case, thus we start from another ansatz to plug in (3.3):

$$
w(s, \tau)=v\left(\frac{s}{\tau}, \tau\right), \quad y=\frac{s}{\tau}, \quad \bar{\tau}=\log (1+\tau) .
$$

By straightforward calculations, we obtain that $v=v(y, \bar{\tau})$ satisfies the following equation

$$
v_{\bar{\tau}}-y v_{y}=\left(v^{m}\right)_{y}+\frac{1}{e^{\bar{\tau}}-1}\left(v^{m}\right)_{y y} .
$$

We are again in a case of asymptotic simplification where the effect of the diffusion term is negligible in the limit. Passing formally to the limit as $\bar{\tau} \rightarrow \infty$ in (5.2), and assuming the limit $v^{*}=v^{*}(y)$ to be stationary in the new variables, we deduce that $v^{*}$ solves the following equation

$$
y v_{y}^{*}=m v^{m-1} v_{y}^{*}
$$

hence, either $v^{*}$ is constant (in some interval), or $v^{*}(y)=(y / m)_{+}^{1 /(m-1)}$ in the complementary part. As the constant part is expected to be equal to the initial value $K$, and undoing the change of variables (5.1), we expect the asymptotic profile for (3.3) in this case to be given by

$$
V(s, \tau)= \begin{cases}0, & \text { if } s \leq 0 \\ {\left[\frac{s}{m \tau}\right]^{1 /(m-1)},} & \text { if } 0<s<m K^{m-1} \tau, \\ K, & \text { if } s \geq m K^{m-1} \tau\end{cases}
$$

Notice that this function is continuous, departing strongly from the profile $W$ introduced in (4.3), which develops a shock curve. Undoing now the transformation (3.1), we arrive to our expected profile

$$
E_{K}(x, t)= \begin{cases}K, & \text { if } 0 \leq|x| \leq e^{-m K^{m-1}(N-2) t} \\ {\left[-\frac{1}{m(N-2)} \frac{\log |x|}{t}\right]^{1 /(m-1)},} & \text { if } e^{-m K^{m-1}(N-2) t}<|x|<1 \\ 0, & \text { if }|x| \geq 1,\end{cases}
$$

which coincides with the one in Theorem 1.5 .

All these calculations are, obviously, formal, showing how the profile $E_{K}$ arises. We are now able to prove rigorously the large-time convergence towards the profile $E_{K}$. 
Proof of Theorem 1.5. Let $u_{0}, u$ as in Theorem 1.5. By the transformation (3.1), we obtain a solution $w$ to (3.3), such that $0 \leq w_{0}(s) \leq K$ for any $s \in \mathbb{R}$, and $\lim _{s \rightarrow \infty} w_{0}(s)=K$, $\lim _{s \rightarrow-\infty} w_{0}(s)=0$. The proof is divided into two big steps: first, we reduce the problem to the case when $w_{0}$ is nondecreasing (or equivalently the initial data $u_{0}$ in initial variables is nonincreasing), and second, we prove the theorem under this extra hypothesis.

\section{Big step A: Reduction to the case of nondecreasing initial data.}

This is based on the following standard result.

Lemma 5.1. Let $w$ be a solution to (3.3) as above such that its initial condition $w_{0}$ is nondecreasing. Then $w(\tau)$ is nondecreasing in $s$ for any $\tau>0$.

We only sketch the proof, see also [8] [Lemma 3.3].

Proof. The general principle is to derive the equation satisfied by the derivative $w_{s}$. As we work with nonnegative functions, it suffices to derive it for any power, in particular for $p=m /(m-1)\left(w^{m-1}\right)_{s}$, which is (4.8). As (4.8) is parabolic, fulfills a comparison principle and $p \equiv 0$ is a solution, it follows that $\left(w^{m-1}(\cdot, \tau)\right)_{s} \geq 0$ for any $\tau>0$, whence $w^{m-1}$ is nondecreasing, hence also $w$.

Suppose that Theorem [1.5 is proved for $w_{0}$ nondecreasing (which is equivalent in the initial variables to $u_{0}$ nonincreasing). Let now a general solution $u$ to (1.1) such that $u_{0}$ satisfies (1.12). Pass again to $w$ solution to (3.3), where $0 \leq w_{0}(s) \leq K$ for any $s \in \mathbb{R}$. Since

$$
\lim _{s \rightarrow-\infty} w_{0}(s)=0, \quad \lim _{s \rightarrow \infty} w_{0}(s)=K,
$$

we can easily find some $w_{0}^{l}, w_{0}^{u}$ which are nondecreasing, satisfying the same limits as in (5.4) and such that

$$
w_{0}^{l}(s) \leq w_{0}(s) \leq w_{0}^{u}(s), \quad \text { for any } s \in \mathbb{R} .
$$

Let $w^{l}, w^{u}$ be the solutions to (3.3) with initial data $w_{0}^{l}, w_{0}^{u}$ respectively. Then, by standard comparison and Lemma 5.1, $w^{l}(\tau), w^{u}(\tau)$ are nondecreasing with respect to $s$ at any time $\tau>0$, and $w^{l}(s, \tau) \leq w(s, \tau) \leq w^{u}(s, \tau)$, for any $(s, \tau) \in \mathbb{R} \times(0, \infty)$. Applying Theorem 1.5 (supposed to be already known for nondecreasing solutions) for $w^{l}, w^{u}$, we get the desired convergence result for $w$, whence for our solution $u$ after undoing the transformation (3.1).

\section{Big step B: Proof for $w_{0}$ nondecreasing.}

From now on, in all this section we work with solutions $w$ to (3.3) with nondecreasing initial data $w_{0}$ as above. We employ the four-step method, which is by now a standard general strategy of proving large time behavior for nonlinear diffusion equations (see e. $\mathrm{g}$. [18, 19, 25]). We have to adapt the technique to the less usual case when the maximum order term will have no effect for large times, as it happens for Eq. (3.3).

Step 1. Rescaling. Define for any $\lambda>0$,

$$
w_{\lambda}(s, \tau)=w(\lambda s, \lambda \tau) .
$$

Then, $w_{\lambda}$ solves the following equation:

$$
\left(w_{\lambda}\right)_{\tau}=\frac{1}{\lambda}\left(w_{\lambda}^{m}\right)_{s s}-\left(w_{\lambda}^{m}\right)_{s} .
$$


This already suggests the asymptotic simplification we expect to get.

Step 2. Uniform estimates. We want to obtain estimates for $w_{\lambda}$ that do not depend on $\lambda$. Since $w_{0} \in L^{\infty}(\mathbb{R})$, we readily get (by standard comparison) that $|w(s, \tau)| \leq\left\|w_{0}\right\|_{\infty}$, whence

$$
\left|w_{\lambda}(s, \tau)\right| \leq\left\|w_{0}\right\|_{\infty}, \quad \text { for any } s \in \mathbb{R}, \tau>0, \lambda>0 .
$$

Moreover, it is proved in [17, Lemma 2.10] that in our conditions,

$$
\left|\left(w^{m}\right)_{s}(s, \tau)\right| \leq \frac{2}{m-1}\left\|w_{0}\right\|_{\infty} t^{-1}
$$

where we can add the modulus in the left-hand side since $\left(w^{m}\right)_{s} \geq 0$, as $w(\cdot, \tau)$ is nondecreasing in $s$ for any $\tau>0$, due to Lemma 5.1. Hence

$$
\left|\left(w_{\lambda}^{m}\right)_{s}(s, \tau)\right|=\lambda\left|\left(w^{m}\right)_{s}(\lambda s, \lambda \tau)\right| \leq \frac{2 \lambda}{m-1}\left\|w_{0}\right\|_{\infty}(\lambda t)^{-1}=\frac{2}{m-1}\left\|w_{0}\right\|_{\infty} t^{-1} .
$$

Both estimates (5.6) and (5.7) are uniform with respect to $\lambda$.

Step 3. Passage to the limit. Due to the previous estimates, we obtain that the family $\left\{w_{\lambda}\right\}$ is uniformly equicontinuous in compact subsets. By Ascoli-Arzelá Theorem, there exists a subsequence (not relabeled) $\left\{w_{\lambda}\right\}$ that converges uniformly on compact sets to some limit profile $w_{\infty}$. We can then pass to the limit in the weak formulation of the equation (5.5). Recall that $w_{\lambda}$ satisfies that

$$
\int_{Q}\left[\Phi_{s}\left(\frac{1}{\lambda}\left(w_{\lambda}^{m}\right)_{s}-w_{\lambda}^{m}\right)-\Phi_{\tau} w_{\lambda}\right] d s d \tau=0, \quad \text { for any } \Phi \in \mathcal{D}(Q), Q=\mathbb{R} \times[0, \infty) .
$$

Since $w_{\lambda} \rightarrow w_{\infty}$ uniformly on compact sets (in particular on the support of $\Phi$ ) and $\left(w_{\lambda}^{m}\right)_{s}$ is bounded uniformly with respect to $\lambda$, we let $\lambda \rightarrow \infty$ to get that

$$
\int_{Q}\left[\Phi_{s} w_{\infty}^{m}+\Phi_{\tau} w_{\infty}\right] d s d \tau=0, \quad \text { for any } \Phi \in \mathcal{D}(Q)
$$

whence $w_{\infty}$ is a weak solution to the conservation law

$$
w_{\infty, \tau}+\left(w_{\infty}^{m}\right)_{s}=0 .
$$

Step 4. Identification of the limit. It remains to show that $w_{\infty}=V$, where $V$ is given in (5.3). To this end, we show first that $w_{\infty}$ takes a Heaviside function as initial trace, that is

$$
\lim _{\tau \rightarrow 0} w_{\infty}(s, \tau)= \begin{cases}K, & \text { if } s \geq 0, \\ 0, & \text { if } s<0,\end{cases}
$$

in the sense of distributions, which is equivalent to prove that

$$
\lim _{\tau \rightarrow 0}\left[\int_{-\infty}^{\infty} w_{\infty}(s, \tau) \Phi(s) d s-K \int_{0}^{\infty} \Phi(s) d s\right]=0,
$$


for any $\Phi \in \mathcal{D}(\mathbb{R})$. For any $\Phi \in \mathcal{D}(\mathbb{R})$, we estimate:

$$
\begin{aligned}
\mid \int_{-\infty}^{\infty} & \left(w_{\lambda}(s, \tau)-w_{\lambda}(s, 0)\right) \Phi(s) d s|=| \int_{-\infty}^{\infty} \int_{0}^{\tau} w_{\lambda, \tau}(s, \theta) \Phi(s) d \theta d s \mid \\
& =\left|\int_{0}^{\tau} \int_{-\infty}^{\infty}\left[\frac{1}{\lambda}\left(w_{\lambda}^{m}\right)_{s s}(s, \theta)-\left(w_{\lambda}^{m}\right)_{s}(s, \theta)\right] \Phi(s) d s d \theta\right| \\
& =\left|\int_{0}^{\tau}\left[\frac{1}{\lambda} \int_{-\infty}^{\infty}\left(w_{\lambda}^{m}\right)(s, \theta) \Phi_{s s}(s) d s+\int_{-\infty}^{\infty}\left(w_{\lambda}^{m}\right)(s, \theta) \Phi_{s}(s) d s\right] d \theta\right| \\
& \leq \int_{0}^{\tau}\left|\int_{-\infty}^{\infty}\left(w_{\lambda}^{m}\right)(s, \theta) \Phi_{s}(s) d s\right| d \theta+\frac{1}{\lambda} \int_{0}^{\tau}\left|\int_{-\infty}^{\infty}\left(w_{\lambda}^{m}\right)(s, \theta) \Phi_{s s}(s) d s\right| d \theta \\
& \leq C_{1}\left\|\Phi_{s}\right\|_{\infty}|\operatorname{supp} \Phi| \tau+\frac{C_{2}}{\lambda}\left\|\Phi_{s s}\right\|_{\infty}|\operatorname{supp} \Phi| \tau=C(\Phi) \tau,
\end{aligned}
$$

where by $|\operatorname{supp} \Phi|$ we understand the Lebesgue measure of the (compact) support of $\Phi$. We have thus proved that

$$
\lim _{\tau \rightarrow 0} \int_{-\infty}^{\infty} w_{\lambda}(s, \tau) \Phi(s) d s=\int_{-\infty}^{\infty} w_{\lambda}(s, 0) \Phi(s) d s,
$$

for any $\Phi \in \mathcal{D}(\mathbb{R})$ and $\lambda>0$, the convergence being uniform with respect to $\lambda$ in any interval $\left[\lambda_{0}, \infty\right)$.

It still remains to prove that

$$
\lim _{\lambda \rightarrow \infty} \int_{-\infty}^{\infty} w_{\lambda}(s, 0) \Phi(s) d s=K \int_{0}^{\infty} \Phi(s) d s,
$$

for any $\Phi \in \mathcal{D}(\mathbb{R})$. To this end, we calculate:

$$
\begin{aligned}
\int_{-\infty}^{\infty} w_{\lambda}(s, 0) \Phi(s) d s & =\left(\int_{-\infty}^{0}+\int_{0}^{\infty}\right) w_{0}(\lambda s) \Phi(s) d s=K \int_{0}^{\infty} \Phi(s) d s \\
& +\int_{0}^{\infty}\left(w_{0}(\lambda s)-K\right) \Phi(s) d s+\int_{-\infty}^{0} w_{0}(\lambda s) \Phi(s) d s .
\end{aligned}
$$

Recall that $\lim _{s \rightarrow \infty} w_{0}(s)=K$ and $\lim _{s \rightarrow-\infty} w_{0}(s)=0$. This implies

$$
\lim _{\lambda \rightarrow \infty} w_{0}(\lambda s) \Phi(s)=0, \quad \text { for any } s<0,
$$

and

$$
\lim _{\lambda \rightarrow \infty}\left(w_{0}(\lambda s)-K\right) \Phi(s)=0, \quad \text { for any } s>0,
$$

with pointwise convergence in both cases. Moreover, since $\Phi$ is compactly supported and $w_{0} \in L^{\infty}(\mathbb{R})$, we can apply the Lebesgue's dominated convergence theorem to find

$$
\lim _{\lambda \rightarrow \infty} \int_{-\infty}^{0} w_{0}(\lambda s) \Phi(s) d s=\lim _{\lambda \rightarrow \infty} \int_{0}^{\infty}\left(w_{0}(\lambda s)-K\right) \Phi(s) d s=0,
$$

to conclude that (5.11) holds. Joining (5.10) and (5.11), we readily get (5.9), as wanted. Thus, $w_{\infty}$ is a generalized (entropy) solution for the conservation law (5.8) with initial condition $K H$. By Kruzhkov's Theorem [15, 23, we find that $w_{\infty} \equiv V$. 
There is a last part in the four-step method, that is, rephrazing the results in terms of the initial variables. We have just proved that

$$
\left|w_{\lambda}(s, \tau)-w_{\infty}(s, \tau)\right| \rightarrow 0 \text { as } \lambda \rightarrow \infty,
$$

uniformly in $(s, \tau)$ in compact subsets of $\mathbb{R} \times[0, \infty)$. We put $\tau=1$, then we relabel $\lambda=\tau$, to get that

$$
\left|w(s \tau, \tau)-w_{\infty}(s, 1)\right| \rightarrow 0 \quad \text { as } \tau \rightarrow \infty,
$$

uniformly for $s$ in compact sets of $\mathbb{R}$. Choosing compacts of the type $[-R, R]$ for $R>0$ large, this is equivalent to say that

$$
|w(s, \tau)-V(s, \tau)| \rightarrow 0 \quad \text { as } \tau \rightarrow \infty,
$$

uniformly for $s \in[-R \tau, R \tau]$. By undoing transformation (3.1) and going back to the initial variables $(x, t)$, we obtain (1.13) in sets of the type $\left\{e^{-R t} \leq|x| \leq e^{R t}\right\}$, for any $R>0$.

Step 5. Behavior at the origin. We go back to initial variables and show that $u(0, t)=$ $K$ for any $t>0$. Assume, by contradiction, that there exists $t_{0}>0$ such that $u\left(0, t_{0}\right)=$ $K_{1}<K$ (if $K_{1}>K$, things are completely similar). Then, we can start the evolution taking $t=t_{0}$ as initial time; by uniqueness, the solution to the Cauchy problem with $v_{0}(x)=u\left(x, t_{0}\right)$ will be $v(x, t)=u\left(x, t+t_{0}\right)$. Applying (1.13) for this $v$, we find that

$$
\lim _{t \rightarrow \infty}\left|u\left(x, t+t_{0}\right)-E_{K_{1}}(x, t)\right|=0,
$$

uniformly in any set of the form $\left\{e^{-R t} \leq|x| \leq e^{R t}\right\}$ for any $R>0$. In particular, choosing $R$ sufficiently large, (the precise condition is $R>m K^{m-1}(N-2)$ ), we reach a contradiction, as in the set $\left\{e^{-R t} \leq|x| \leq e^{R t}\right\}$, the two profiles $E_{K}$ and $E_{K_{1}}$ are essentially different: $\lim _{t \rightarrow \infty}\left\|E_{K}(t)-E_{K_{1}}(t)\right\|_{\infty}=K-K_{1}>0$. Hence $u(0, t)=K$ for any $t>0$.

Step 6. Uniform convergence in the whole space. We have shown up to now that (1.13) holds true uniformly in sets of the form

$$
\left\{e^{-R t} \leq|x| \leq e^{R t}\right\}, \quad \text { for any } R>0 .
$$

In order to extend the uniform convergence to the whole $\mathbb{R}^{N}$, we essentially use the fact that $u(t)$ is nonincreasing for any $t>0$. Let some $\varepsilon_{0}>0$ fixed. Then, for any $t>0$, we have $u\left(\varepsilon_{0}, t\right) \leq u(x, t)=u(|x|, t) \leq u(0, t)=K$, that is, $u(\cdot, t)$ is uniformly Cauchy in $\left[0, \varepsilon_{0}\right]$, whence the uniform convergence is extended up to the origin. A similar argument holds for the tail part $\left\{|x| \geq e^{R t}\right\}$ closing the proof.

\section{Asymptotic convergence for general solutions}

We are now ready to prove our results for non-radially symmetric solutions, which are Theorem [1.6] and Corollary 1.4.

Proof of Theorem 1.6. Let $u$ be a solution to (1.1) with initial condition $u_{0}$ satisfying (1.5), (1.12) and (1.15), with $u_{0}(0)=K>0$. We define the following radially symmetric functions:

$u_{0}^{ \pm}: \mathbb{R}^{N} \mapsto[0, \infty), \quad u_{0}^{-}(r)=\inf \left\{u_{0}(x):|x|=r\right\}, \quad u_{0}^{+}(r)=\sup \left\{u_{0}(x):|x|=r\right\}, \quad r=|x|$. 
It is obvious that $u_{0}^{-}(x) \leq u_{0}(x) \leq u_{0}^{+}(x)$, for any $x \in \mathbb{R}^{N}$, and $u_{0}^{-}(0)=u_{0}^{+}(0)=K$. Moreover, both $u_{0}^{-}$and $u_{0}^{+}$are continuous at $x=0$ and belong to $L^{\infty}\left(\mathbb{R}^{N}\right)$, as $0<$ $\left\|u_{0}^{-}\right\|_{\infty}=\left\|u_{0}\right\|_{\infty}=\left\|u_{0}^{+}\right\|_{\infty}=K$.

It remains to check that $u_{0}^{-}, u_{0}^{+} \in L_{2}^{1}\left(\mathbb{R}^{N}\right)$. We only have to check this in sets that are close to $x=0$ and to infinity. Since $u_{0}(0)=K$ and it is continuous, there exists $r>0$ such that $K-1<u_{0}(x)<K+1$, for any $x \in B(0, r)$. By definition, we also have $K-1<u_{0}^{-}(x) \leq u_{0}^{+}(x)<K+1$, for any $x \in B(0, r)$, hence

$$
0<\int_{B(0, r)}|x|^{-2} u_{0}^{-}(x) d x \leq \int_{B(0, r)}|x|^{-2} u_{0}^{+}(x) d x \leq(K+1) \int_{B(0, r)}|x|^{-2} d x<\infty,
$$

as we are in dimension $N \geq 3$. Concerning sets that are "close to infinity", we deduce from (1.15) that

$$
u_{0}^{-}(x), u_{0}^{+}(x) \leq|x|^{2-N-\delta}, \quad \text { for any } x \in \mathbb{R}^{N} \backslash B(0, R),
$$

whence

$$
0<\int_{\mathbb{R}^{N} \backslash B(0, R)}|x|^{-2} u_{0}^{-}(x) d x \leq \int_{\mathbb{R}^{N} \backslash B(0, R)}|x|^{-2} u_{0}^{+}(x) d x \leq \int_{\mathbb{R}^{N} \backslash B(0, R)}|x|^{-N-\delta} d x<\infty .
$$

This, together with the uniform boundedness in the compact set $\overline{B(0, R)} \backslash B(0, r)$, show that $u_{0}^{-}, u_{0}^{+} \in L_{2}^{1}\left(\mathbb{R}^{N}\right)$. By Theorem 2.1. there exist $u^{-}, u^{+}$solutions to Eq. (1.1) with initial data $u_{0}^{-}, u_{0}^{+}$respectively; as Eq. (1.1) is rotationally invariant, $u^{-}$and $u^{+}$are radially symmetric and they fulfill the assumptions in Theorem 1.5. It follows that

$$
\lim _{t \rightarrow \infty}\left|u^{-}(x, t)-E_{K}(x, t)\right|=\lim _{t \rightarrow \infty}\left|u^{+}(x, t)-E_{K}(x, t)\right|=0,
$$

uniformly in $\mathbb{R}^{N}$. By standard comparison, $u^{-}(x, t) \leq u(x, t) \leq u^{+}(x, t)$ for any $(x, t) \in$ $\mathbb{R}^{N} \times[0, \infty)$, and the conclusion of Theorem 1.6 follows in an obvious way. Proof of Theorem 1.6 under conditions in Remark 1.7 is totally similar.

Proof of Corollary 1.4. Define $u_{0}^{ \pm}$and $u^{ \pm}$as above. Then $u^{+}$and $u^{-}$are radially symmetric solutions satisfying the conditions in Theorem 1.1, and $u^{-}$is nontrivial due to the condition (1.11). By Theorem 1.3, $\left\|u^{ \pm}(t)\right\|_{\infty}$ decay exactly with rate $t^{-1 / m}$. Since by comparison, $u^{-}(x, t) \leq u(x, t) \leq u^{+}(x, t)$ for any $(x, t) \in \mathbb{R}^{N} \times[0, \infty)$, the same decay rate holds true for $\|u(t)\|_{\infty}$.

Notice that we cannot have a more precise asymptotic convergence result for general solutions $u$ to Eq. (1.1) through this method, since the limit profiles depend essentially on the mass $M_{u_{0}}$, and there is no obvious connection between $M_{u_{0}}$ and $M_{u_{0}^{ \pm}}$.

\section{Further results, extensions and applications}

In this final section, we gather some facts that, at a formal level, extend or apply our analysis to other equations or different conditions. 


\subsection{A different critical density: $\gamma=N-(N-2) / m$}

In a previous work [9, Theorem 2.1], it has been noticed that Eq. (1.1) can be mapped, at the level of radially symmetric solutions, into a similar equation presenting a different, but also critical, density. More precisely, at a formal level, given a radially symmetric solution $u$ to Eq. (1.1), we define

$$
\tilde{u}(r, t):=r^{(2-N) / m} u\left(r^{-1}, t\right), \quad r=|x|,
$$

and find $([9])$ that $\tilde{u}$ is a radially symmetric solution to

$$
|x|^{-\gamma_{2}} \tilde{u}_{t}=\Delta \tilde{u}^{m}, \quad \gamma_{2}=N-\frac{N-2}{m} .
$$

The critical behavior of the exponent $\gamma_{2}$ has been analyzed in previous works as [11, 9, Subsection 3.3]. In particular, the theory developed in [11, Section 6] holds exactly for $\gamma<\gamma_{2}$ but fails to hold for this borderline case.

Taking into account (7.1) and our results, and refraining from performing a rigorous analysis of Eq. (7.2), we can give some ideas about what is expected to happen with its solutions. The profiles $E_{K}$ and $F$ transform into

$$
\tilde{E}_{K}(x, t)= \begin{cases}0, & \text { if }|x| \leq 1, \\ |x|^{(2-N) / m}\left[\frac{1}{m(N-2)} \frac{\log |x|}{t}\right]_{+}^{1 /(m-1)}, & \text { if } 1<|x|<e^{m(N-2) K^{m-1} t}, \\ |x|^{(2-N) / m} K, & \text { if }|x| \geq e^{m(N-2) K^{m-1} t}\end{cases}
$$

and

$$
\tilde{F}(x, t)= \begin{cases}t^{-1 / m}|x|^{(2-N) / m}\left[\frac{\log |x| t^{-1 / m}}{m(N-2)}\right]_{+}^{1 /(m-1)}, & \text { if } 0 \leq|x|<e^{k t^{1 / m}} \\ 0, & \text { if }|x| \geq e^{k t^{1 / m}}\end{cases}
$$

the first being supported in the interval $[1, \infty)$ and the second in $\left[1, e^{k t^{1 / m}}\right]$.

Moreover, there exists an explicit family of self-similar solutions to (17.2), that is obtained starting from the logarithmic Barenblatt solutions to (1.1) given in (1.16); by mapping them via (7.1), we deduce explicit solutions to (7.2) having the self-similar form

$$
\tilde{B}_{D}(x, t)=t^{-1 / m} U_{D}\left(|x| t^{1 /(m-1)(N-2)}\right), \quad U_{D}(\xi)=\xi^{(2-N) / m}\left[D+\frac{1}{m(N-2)} \log \xi\right]_{+}^{1 /(m-1)},
$$

for any $D>0$. Up to our knowledge, the self-similar functions in (7.3) are new. Notice that they have a time decay $t^{-1 / m}$, but also a backward evolution of the support, that is,

$$
\operatorname{supp} \tilde{B}_{D}=\left\{(x, t) \in \mathbb{R}^{N} \times(0, \infty):|x|>e^{-D m(N-2)} t^{-1 /(m-1)(N-2)}\right\} .
$$

By applying transformation (7.1) to our results, it is expected then that $\tilde{E}_{K}$ be the family of general asymptotic profiles of solutions $\tilde{u}$ to (7.2) decaying at infinity exactly like $K|x|^{(2-N) / m}$, with $\lim _{|x| \rightarrow 0} \tilde{u}(x, t)=0$ while $\tilde{F}$ is the general asymptotic profile of solutions $\tilde{u}$ to (7.2) having the same behavior at the origin but a weaker decay as $|x| \rightarrow \infty$. 
Since (7.1) is an inversion, a stronger role will play our new explicit solution in (7.3), with respect to large-time behavior in inner regions, close to the origin. Indeed, by rephrazing Theorem 1.9 for $\tilde{u}$ and performing the changes to self-similar variables as in (7.3), one gets the following expected asymptotic convergence for $\gamma=\gamma_{2}$ :

$$
\lim _{t \rightarrow \infty}\left|\tilde{u}(x, t)-\tilde{B}_{D}(x, t)\right|=0
$$

uniformly in sets of the form $|x| \leq K t^{-1 /(m-1)(N-2)}$ for any $K>0$, that is, in small inner sets shrinking to the origin as $t \rightarrow \infty$. This result will be interesting from the point of view of explaining the influence of the singularity, which is the most important feature of (7.2).

A rigorous study of this critical case will be left for future work.

\subsection{Some open questions}

Related to the analysis performed in the present work, we leave below a list of, in our opinion, interesting questions that can be addressed in future developments of the subject.

1. The inner behavior for nonsingular densities. As explained in the Introduction, there was an important mathematical interest for the study of (1.2) with densities $\varrho$ that are regular at $x=0$ and have $\varrho(x) \leq C|x|^{-\gamma}$ as $|x| \rightarrow \infty$, see [20, 21, 22, 11, 18. In particular, it has been shown that the large-time behavior is strongly related to fundamental solutions to our Eq. (1.1). The density $\gamma=2$ is critical [1] and the authors of [18] prove the largetime behavior in outer sets, similar to our Theorem 1.9. The problem of understanding the inner behavior is left open. We don't know whether our results in the present paper can give more light on this subject, as we analyze the inner behavior for Eq. (1.1), but the difference between densities $\varrho$ as considered in [18, Subsection 2.1] and our singular $\varrho(x)=|x|^{-2}$ might play an essential role.

2. Removing a condition in Theorems 1.5 and 1.6. One would like to eliminate the condition $0 \leq u_{0}(x) \leq K$ from the statement of Theorems 1.5, 1.6 and prove them for general $u_{0} \in L^{\infty}\left(\mathbb{R}^{N}\right)$; this would allow functions that can have a peak in $\{0<|x|<\infty\}$ but still satisfying

$$
\lim _{|x| \rightarrow \infty} u_{0}(x)=0, \quad u_{0}(0)=K>0 .
$$

We conjecture that Theorem 1.5 and Theorem 1.6 remain true in this case, that is, there exists some mechanism in the Eq. (1.1) forcing a maximum attained at some point different from the origin to go down in time. This is confirmed by the numerical experiments, as Figure 3 suggests.

We can see that starting from a radially symmetric initial data with a maximum attained for some $|x|=r(0) \in(0, \infty)$, and letting for any $t>0$

$$
u(r(t), t)=\max \left\{u(|x|, t): x \in \mathbb{R}^{N}\right\},
$$

then $u(r(t), t)$ decreases with $t$, but at the same time $r(t) \rightarrow 0$. Thus, the slope of the graph in $[0, r(t)]$ may be quite big for $t>0$ very large, which shows that, even if we expect the same result as in Theorem 1.5 to hold true, our technique essentially based on a boundedness of the derivative cannot be used. It seems that we need some different ideas. 


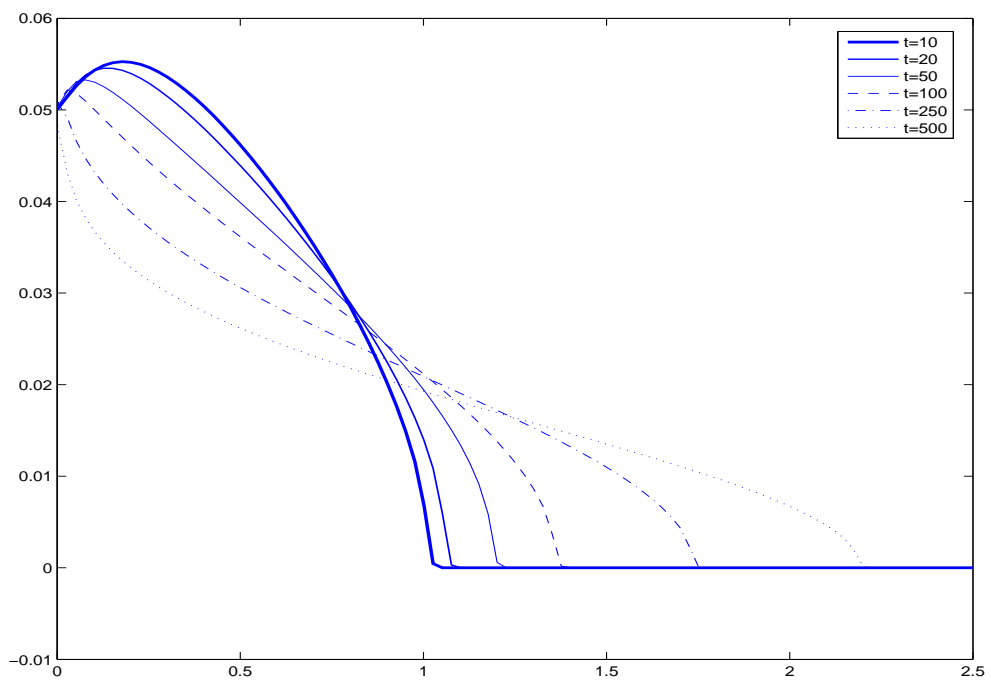

Figure 3: Evolution of a solution with maximum attained outside the origin.

3. Large time behavior for general solutions in Theorem 1.3, While for solutions to (1.1) with initial data $u_{0}(0)=K>0$, our analysis holds true for general (not necessarily radially symmetric) solutions, this problem remains open in the case when $u_{0}(0)=0$. Notice that a similar proof as that of Theorem 1.6 does not hold, due to the essential dependence of the limit profile on some weighted mass of the initial condition. As suggested in [10, one might expect a negative answer.

AcKnowledGements. R. I. partially supported by the Spanish project MTM201231103. A. S. partially supported by the Spanish project MTM2011-25287. Part of this work has been done during visits by A. S. to the Departamento de Análisis Matemático of the Universidad de Valencia.

\section{References}

[1] Ph. Benilan, H. Touré, Sur l'equation generale $u_{t}=a\left(\cdot, u, \varphi(\cdot, u)_{x}\right)_{x}+v$ dans $L^{1}$, Ann. Inst. H. Poincaré Anal. Non Lineaire, 12 (1995), 727-761.

[2] J. I. Diaz, R. Kersner, On a nonlinear degenerate parabolic equation in infiltration or evaporation through a porous medium, J. Differential Equations, 69 (1987), 368-403.

[3] D. Eidus, The Cauchy problem for the nonlinear filtration equation, J. Differential Equations, 84 (1990), 309-318.

[4] D. Eidus, S. Kamin, The filtration equation in a class of functions decreasing at infinity, Proc. Amer. Math. Society, 120 (1994), no. 3, 825-830. 
[5] M. Escobedo, J. L. Vázquez, E. Zuazua, Asymptotic behavior and source-type solutions for a diffusion-convection equation, Arch. Rational Mech. Anal, 124 (1993), 43-65.

[6] B. H. Gilding, Improved theory for a nonlinear degenerate parabolic equation, Ann. Scuola Norm. Sup. Pisa, 16 (1989), 165-224.

[7] R. E. Grundy, C. J. van Duijn, C. N. Dawson, Asymptotic profiles with finite mass in one-dimensional contaminant transport through porous media: the fast reaction case, Quart. J. Mech. Appl. Math., 47 (1994), 69-106.

[8] R. Iagar, Ph. Laurençot, J. L. Vázquez, Asymptotic behavior of a nonlinear parabolic equation with gradient absorption and critical exponent, Interfaces Free Bound., 13 (2011), no. 2, 271-295.

[9] R. Iagar, G. Reyes, A. Sánchez, Radial equivalence of nonhomogeneous nonlinear diffusion equations, Acta Appl. Math., 123 (2013), 53-72.

[10] R. Iagar, A. Sánchez, Asymptotic behavior for the heat equation in nonhomogeneous media with critical density, Nonl. Anal., 89 (2013), 24-35.

[11] S. Kamin, G. Reyes, J. L. Vázquez, Long time behavior for the inhomogeneous PME in a medium with rapidly decaying density, Discrete Contin. Dyn. Syst. 26 (2010), no. $2,521-549$.

[12] S. Kamin, P. Rosenau, Propagation of thermal waves in an inhomogeneous medium, Comm. Pure Appl. Math, 34 (1981), no. 6, 831-852.

[13] S. Kamin, P. Rosenau, Nonlinear thermal evolution in an inhomogeneous medium, J. Math. Phys., 23 (1982), no. 7, 1385-1390.

[14] S. Kamin, P. Rosenau, Thermal waves in an absorbing and convecting medium, Phys. D., 8 (1983), no. 1-2, 273-283.

[15] S. N. Kruzhkov, First order quasilinear equations in several independent variables, Math. of the USSR-Sbornik 10 (1970), no. 2, 217-243.

[16] Ph. Laurençot, F. Simondon, Source-type solutions to porous medium equations with convection, Commun. Appl. Anal., 1 (1997), no. 4, 489-502.

[17] $\mathrm{Ph}$. Laurençot, F. Simondon, Long-time behavior to porous medium equations with convection, Proc. Roy. Soc. Edinburgh Sect. A, 128 (1998), no. 2, 315-336.

[18] E. S. Nieto, G. Reyes, Asymptotic behavior of the solutions of the inhomogeneous porous medium equation with critical vanishing density, Commun. Pure Appl. Anal., 12 (2013), no. 2, 1123-1139.

[19] G. Reyes, Asymptotic behavior in convection-diffusion processes, Nonl. Anal., 37 (1999), no. 3, 301-318.

[20] G. Reyes, J. L. Vázquez, The Cauchy problem for the inhomogeneous porous medium equation, Network Heterog. Media 1 (2006), no. 2 337-351. 
[21] G. Reyes, J. L. Vázquez, The inhomogeneous PME in several space dimensions. Existence and uniqueness of finite energy solutions, Commun. Pure Appl. Anal., 7 (2008), no. $6,1275-1294$.

[22] G. Reyes, J. L. Vázquez, Long time behavior for the inhomogeneous PME in a medium with slowly decaying density, Commun. Pure Appl. Anal., 8 (2009), no. 2, 493-508.

[23] D. Serre, Systems of conservation laws 1: hiperbolicity, entropies, shock waves, third ed., Cambridge Univ. Press, 2003.

[24] A. F. Tedeev, The interface blow-up phenomenon and local estimates for doubly degenerate parabolic equations, Appl. Anal., 86 (2007), no. 6, 755-782.

[25] J. L. Vázquez, Asymptotic behavior for the porous medium equation posed in the whole space. Dedicated to Philippe Benilan, J. Evol. Equ. 3 (2003), no. 1, 67-118. 